\title{
ERRATUM
}

\section{Erratum: Independent associations of total and high molecular weight adiponectin with cardiometabolic risk and surrogate markers of enhanced early atherogenesis in black and white patients with rheumatoid arthritis: a cross-sectional study}

\author{
Patrick H Dessein ${ }^{*}$, Angela J Woodiwiss ${ }^{1}$, Gavin R Norton ${ }^{1}$, Linda Tsang ${ }^{2}$ and Ahmed Solomon ${ }^{3}$ \\ See related research by Dessein et al., http://arthritis-research.com/content/15/5/R128
}

After publication of our recent article [1], it has been brought to our attention that the adiponectin concentrations are mislabelled as nanograms/millilitre $(\mathrm{ng} / \mathrm{ml})$. The total and high molecular weight concentrations were measured in micrograms/millilitre $(\mu \mathrm{g} / \mathrm{ml})$.

\footnotetext{
Author details

'Cardiovascular Pathophysiology and Genomics Research Unit, School of Physiology, Faculty of Health Sciences, University of the Witwatersrand, Private Bag 3, Wits 2050, Johannesburg, South Africa. ${ }^{2}$ Milpark Hospital, P. O. Box 91155, Auckland Park 2006, Johannesburg, South Africa. ${ }^{3}$ Department of Rheumatology, Charlotte Maxeke Johannesburg Academic Hospital, Faculty of Health Sciences, University of the Witwatersrand, Private Bag 3, Wits 2050, Johannesburg, South Africa.
}

Published online: 20 December 2014

\section{Reference \\ 1. Dessein H, Woodiwiss A, Norton GR, Tsang L, Solomon A: Independent associations of total and high molecular weight adiponectin with cardiometabolic risk and surrogate markers of enhanced early atherogenesis in black and white patients with rheumatoid arthritis: a cross-sectional study. Arthritis Res Ther 2013, 15:R128.}

\section{doi:10.1186/s13075-014-0503-3}

Cite this article as: Dessein et al:: Correction: Independent associations of total and high molecular weight adiponectin with cardiometabolic risk and surrogate markers of enhanced early atherogenesis in black and white patients with rheumatoid arthritis: a cross-sectional study. Arthritis Research \& Therapy 2014 16:503.

\footnotetext{
* Correspondence: Dessein@telkomsa.net

${ }^{1}$ Cardiovascular Pathophysiology and Genomics Research Unit, School of Physiology, Faculty of Health Sciences, University of the Witwatersrand, Private Bag 3, Wits 2050, Johannesburg, South Africa
} 